\title{
A rare case report of transient pancytopenia in a patient with coronavirus disease 2019
}

\author{
Vallish Shenoy ${ }^{1}$, Chandra Shekhar $N^{1}$, Preetham Raj TS ${ }^{2}$ \\ From Junior Resident, ${ }^{1}$ Department of Internal Medicine and ${ }^{2}$ Pathology, Bangalore Medical College and Research Institute, KR Road, Bengaluru, \\ Karnataka, India
}

\begin{abstract}
Severe acute respiratory syndrome coronavirus 2 (SARS-CoV-2) is responsible for coronavirus disease 2019 (COVID-19) infection. The ongoing pandemic of COVID-19 has sought the attention of clinicians and researchers to investigate and describe its various pathological aspects. Infection with SARS-CoV-2 and the resulting COVID-19 can affect virtually any organ of the human body, including the bone marrow which, in turn, causes pancytopenia. Diagnosing pancytopenia in the setting of COVID-19 infection is challenging and requires ruling out all other potential causes of pancytopenia. Herein, we report a rare case of severe pancytopenia secondary to COVID-19 infection that developed severe transient pancytopenia which improved with conservative management.
\end{abstract}

Key words: Coronavirus disease 2019, Severe acute respiratory syndrome coronavirus-2, Pancytopenia, Cytokine storm

$\mathrm{E}$ xperimental research findings indicate that exaggerated immune response and a strong cytokine storm which may include high levels of granulocyte-macrophage colony-stimulating factor and interleukin-6 (IL-6) are activated in severe coronavirus disease 2019 (COVID-19) [1]. This can lead to a clinical presentation of pancytopenia. Severe acute respiratory syndrome coronavirus-2 (SARSCoV-2), the etiologic agent of the COVID-19 pandemic, has spread rapidly worldwide since December 2019. Although COVID-19 primarily attacks the respiratory system, new clinical manifestations emerge every day and should not be neglected, even the most innocuous ones. SARS-CoV-2 viremia and pancytopenia from the infiltration of bone marrow constitute a rare COVID-19 manifestation. With regard to blood cells, many changes were described, most commonly including lymphopenia, leukocytosis, neutrophilia, and thrombocytopenia [1]. On very rare occasions, the patients might develop neutropenia or pancytopenia [2]. Therapeutic approaches for pancytopenia in the context of COVID-19 are unknown and the use of granulocyte colony-stimulating factor (GCSF) is controversial due to concern for increased morbidity and mortality [3]. The case report described here presents a patient with COVID-19 that developed severe transient pancytopenia which improved with conservative management.

\section{Access this article online}

Received - 25 April 2021

Initial Review - 11 May 2021

Accepted - 21 June 2021

DOI: $10.32677 / \mathrm{IJCR} .2021 . v 07.107 .003$

\section{CASE REPORT}

A 35-year-old male presented to our emergency department with complaints of fever, cough, and malaise for 5 days, shortness of breath for 2 days, and extreme fatigue and petechial rash on lower limbs for 1 day. There was no history of previous similar episodes or any family history suggestive of malignancy or bone marrow failure.

On general examination, the patient was well-built and conscious, febrile, tachypneic with a respiratory rate of 24/ min, resting pulse was $116 / \mathrm{min}$ and regular, blood pressure was $110 / 60 \mathrm{mmHg}$, the body temperature was $102.1^{\circ} \mathrm{F}$, and oxygen saturation $\mathrm{SpO}_{2}$ of $91 \%$ at room air and $99 \%$ on $2 \mathrm{~L}$ of oxygen through nasal prongs. Conjunctival pallor with lower limb petechiae was noted. Cardiovascular examination revealed tachycardia but no gallop; mild fine crepitations with decreased breath sounds were noted at the basal lung fields on respiratory system examination with per abdominal examination unremarkable with no organomegaly.

Initial laboratory workup done in our hospital revealed hemoglobin of $10.1 \mathrm{~g} / \mathrm{dl}$, total leukocyte count of $2260 / \mathrm{mm}^{3}$ (differential count showing lymphopenia and mild neutropenia with relative monocytosis), and platelet count of $10,000 / \mathrm{mm}^{3}$ with mean platelet volume of 7.3. Peripheral smear showed normocytic normochromic anemia with occasional band forms with a decrease in both white blood cells (WBCs) and platelets (Fig. 1). Reticulocyte count (corrected) was $0.98 \%$. Erythrocyte

Correspondence to: Dr. Vallish Shenoy, Department of Medicine, Bangalore Medical College and Research Institute, KR Road, Bengaluru, Karnataka, India. E-mail: shenvoy55@gmail.com

(C) 2021 Creative Commons Attribution-NonCommercial 4.0 International License (CC BY-NC-ND 4.0). 
sedimentation rate was $50 \mathrm{~mm}$ in the $1^{\text {st }} \mathrm{h}$. Viral serology was negative. The coagulation profile was slightly deranged with activated partial thromboplastin time which was 25.2 and D-dimer was $1.29 \mathrm{mcg} / \mathrm{mL}$ (Table 1). The liver and kidney function tests were within normal limits. Direct and indirect Coombs tests were negative. Rheumatoid factor and antinuclear factor were negative. Viral tests for hepatitis $\mathrm{B}, \mathrm{HepC}$, human immunodeficiency virus (HIV), cytomegalovirus (CMV), and Epstein-Barr virus (EBV) were negative. The electrocardiogram showed a regular sinus rhythm of $116 \mathrm{bpm}$ and QT at $410 \mathrm{~mm}$ without repolarization disorders. The echocardiography showed normal ventricular chambers with a systolic ejection fraction of $60 \%$, no valve abnormality, and no signs of pulmonary hypertension or acute cor pulmonale. The patient was then tested for COVID-19 on a nasopharyngeal sample using the reverse transcriptionpolymerase chain reaction (RT-PCR) technique, which came out to be positive.

Chest radiography showed bilateral diffuse lower zone reticular and consolidatory changes with increased bronchovascular markings (Fig. 2) with high-resolution computed tomography (CT) thorax showing multiple subpleural, lobular, and peribronchial

Table 1: Serial CBC and inflammatory markers showing progressive improvement in the cell lines, thus proving transient pancytopenia

\begin{tabular}{|c|c|c|c|}
\hline Parameters & Day 1 & Day 5 & Day 10 \\
\hline Hemoglobin (g/dL) & 9.8 & 10.4 & 10.9 \\
\hline Total count (cells/mm³) & 2,260 & 4.800 & 6,200 \\
\hline Platelet count $\left(\right.$ cells $\left./ \mathrm{mm}^{3}\right)$ & 10,000 & 97,000 & $1,57,000$ \\
\hline Prothrombin time/INR & $12.1 / 1.18$ & $12.1 / 1.18$ & $12.0 / 1.14$ \\
\hline D-dimer $(\mathrm{mcg} / \mathrm{mL})$ & 1.29 & 0.99 & 0.62 \\
\hline C-reactive protein $(\mathrm{mg} / \mathrm{L})$ & 97.23 & 56.42 & 21.11 \\
\hline Serum ferritin $(\mathrm{ng} / \mathrm{ml})$ & 866.48 & 500.12 & 466.90 \\
\hline Lactate dehydrogenase (U/L) & 680 & 498 & 312 \\
\hline Interleukin-6 (pg/ml) & 60.43 & 21.6 & 7.45 \\
\hline
\end{tabular}

CBC: Complete blood count

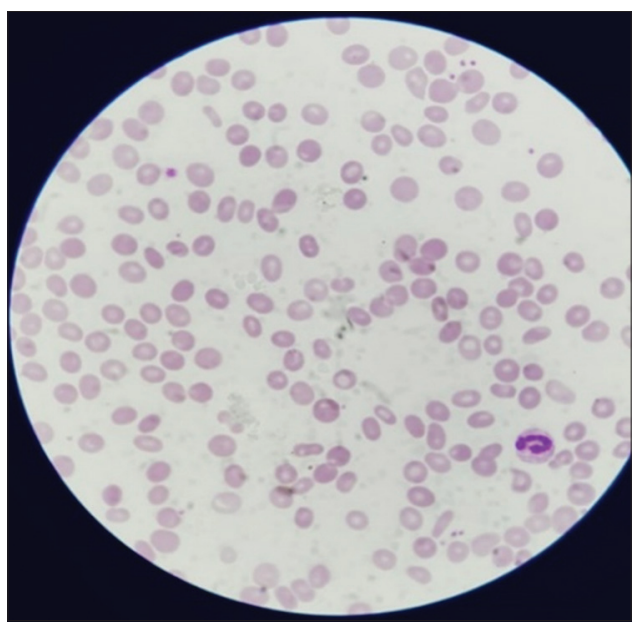

Figure 1: Peripheral smear showing normocytic normochromic anemia with occasional band forms (arrow), pencil cells with anisopoikilocytosis with decrease in both white blood cells and platelets suggestive of pancytopenia areas of ground-glass opacities, with few areas of consolidation in the background of interlobular septal thickening, noted in lower segments of lung fields with CT severity score of 17/25 (Fig. 3). Ultrasound abdomen and pelvis were unremarkable. Moreover, blood culture, urine culture, sputum Gram stain, and culture were negative with normal blood procalcitonin levels. Serial blood counts and inflammatory markers done on consecutive days showed improvement in all the blood cell lines with improvement in the general condition of the patient (Table 1).

The patient was then transferred to the COVID-19 isolation high dependency unit, put on high-flow nasal oxygen, and tapered to room air as he improved. He was given antipyretic paracetamol depending on febrile episodes along with Tab. azithromycin $500 \mathrm{mg}$ per oral once a day and was also started on Inj. enoxaparin $60 \mathrm{mg}$ subcutaneous twice a day, Inj. methylprednisolone $60 \mathrm{mg}$ intravenous once a day, and Inj. remdesivir $200 \mathrm{mg}$ intravenous infusion on day 1 followed by $100 \mathrm{mg}$ intravenous infusion from day 2 to 5 , in concurrence with the local COVID-19 protocol. Due to the low platelets and widespread lower limb petechiae, he received eight units of platelet transfusion and was conservatively managed.

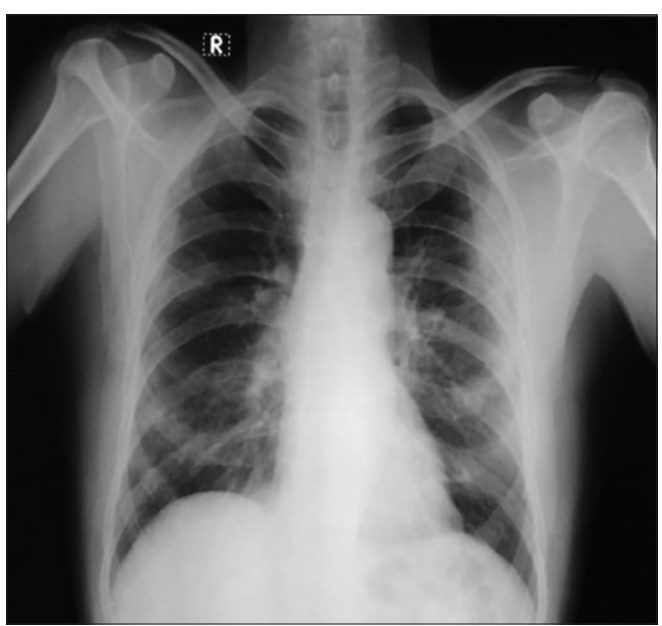

Figure 2: Chest radiography showing bilateral, diffuse, lower zone reticular, and consolidatory changes with increased bronchovascular markings

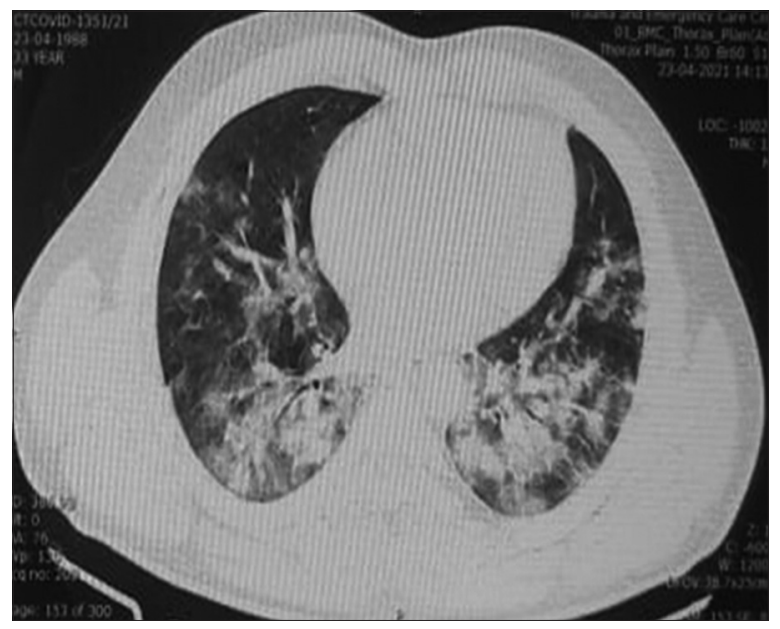

Figure 3: Patient's high-resolution computed tomography showing predominantly basal, posterior and subpleural ground-glass opacities, and consolidation with normal cardiac parameters 
In view of improvement in all the blood cell lines and inflammatory markers on consecutive days with improvement in the general condition of the patient, he was discharged on day 11 with his repeat nasopharyngeal RT-PCR for COVID-19 being negative and was asked to follow-up and report to us in case of recurrence of symptoms for bone marrow examination at a later date.

\section{DISCUSSION}

Pancytopenia is a common hematological condition often encountered in day-to-day clinical practice. It is defined as hemoglobin of $<10 \mathrm{~g} / \mathrm{dl}$, WBC $<4000 / \mathrm{mm}$, and platelets $<100,000 / \mathrm{mm}$ [1]. Severe pancytopenia is defined as absolute neutrophil count $<500 / \mathrm{cmm}$, platelet count $<20,000 / \mathrm{mm}$, and corrected reticulocyte count $<1 \%$ [4].

Clinical presentations related to pancytopenia can be attributed to anemia, leukopenia, and/or thrombocytopenia. Anemia presents with fatigue, breathlessness, and cardiac symptoms. Neutropenia presents with febrile illness due to increased susceptibility to infections. Thrombocytopenia may present with mucocutaneous bleed or bruising. However, the bleeding tendency is seen when platelets are $<10,000$ [5]. Pancytopenia patients can be relatively stable on presentation, however, can develop overwhelming sepsis without any focal signs of infection, with malaise and fever being the only clinical features. Hence, it is imperative to find the cause and treat accordingly [6].

The underlying mechanisms include a decrease in hematopoietic cell production, marrow replacement by abnormal cells, suppression of marrow growth and differentiation, ineffective hematopoiesis with cell death, peripheral destruction of cells, antibody-mediated sequestration, or destruction of cells and trapping of cells in a hypertrophied and overactive reticuloendothelial system [7]. It often represents a diagnostic challenge, mainly because patients are increasingly complex, often having multiple potential confounding (and contributing) comorbidities and medications [8].

Bone marrow suppression can be seen in various viral infections including EBV, HIV, CMV, and parvovirus B19 [9]. After viral infection, an antigenic epitope on myelocytes could be exposed, leading to the production of autoantibody and the destruction of blood cells. The angiotensin-converting enzyme 2 receptor, the target of the SARS-CoV-2, has been identified in bone marrow albeit at a low level [10]. Therefore, it is possible that direct infection of myelocytes could lead to bone marrow suppression. Another key feature of COVID-19 is an elevated level of pro-inflammatory cytokines. It is well-known that certain cytokines, such as interferons and tumor-necrosis factor- $\alpha$, can affect hematopoietic stem cells and impair hematopoiesis [11]. In addition, the lung has been recently identified as a site for platelet biogenesis and a reservoir for hematopoietic progenitors. With SARS-CoV-2 infection of the bone marrow and lung injury, it is possible that the destruction of lung hematopoietic progenitors could also contribute to pancytopenia [12].
Furthermore, CT scores exhibit a potential role in predicting the outcome of SARS-CoV patients. CT score is highly correlated with laboratory findings and disease severity and might be beneficial to speed up diagnostic workflow in symptomatic cases. A CT score of $\geq 18$ has shown to be highly predictive of patient's mortality in short-term follow-up. The CT score noted is 17/25 in our case and it falls into the moderate disease severity category with a sufficiently good prognosis [13].

In our case, we hypothesize that after ruling out most of the causes of pancytopenia, the transient pancytopenia which improved progressively on serial blood counts was due to SARS-CoV-2 infection resulting in sudden maturation arrest and decrease in all the three cell lines and since the patient also had lung injury, it added to the severity of pancytopenia as the lung is one of the sites of extramedullary hematopoiesis. A recent study done by Hersby et al. showed that COVID-19-associated pancytopenia can be self-limiting and does not necessarily warrant bone marrow biopsy for the purpose of SARS-CoV-2 diagnostics [14]. In our case too, the pancytopenia was transient and self-limiting which improved with a decrease in the viral load not warranting any further workup with a good overall prognosis.

\section{CONCLUSION}

This case report highlights the importance of understanding the temporal relationship between COVID-19 and pancytopenia. This case report is also extremely relevant since it illustrates the complexity of the differential diagnosis of pancytopenia and how this disorder can result from a combination of multiple etiologic factors rather than just one. In summary, the clinicians should be aware of the atypical presentation of COVID-19 (transient pancytopenia) which usually does not require any extra workup and is self-limiting with a good prognosis.

\section{REFERENCES}

1. Frater JL, Zini G, d'Onofrio G, Rogers HJ. COVID-19 and the clinical hematology laboratory. Int J Lab Hematol 2020;42 Suppl 1:11-8.

2. Spencer HC, Wurzburger R. COVID-19 presenting as neutropenic fever. Ann Hematol 2020;99:1939-40.

3. Nawar T, Morjaria S, Kaltsas A, Patel D, Perez-Johnston R, Daniyan AF, et al. Granulocyte-colony stimulating factor in COVID-19: Is it stimulating more than just the bone marrow? Am J Hematol 2020;95:E210-3.

4. Watson, Henry G, editors. Blood disease. In: Davidson's Principles and Practice of Medicine. Amsterdam: Elsevier; 2013. p. 989-1056.

5. Deshpande SV, Godbole VY, Asher AD. Pancytopenia: The perspective from Western Gujarat, India. Int J Adv Med 2019;6:731-7.

6. Garg AK, Agarwal AK, Sharma GD. Pancytopenia: Clinical Approach. Ch. 95. 2017. p. 450-4. Available from: http://www.apiindia.org/pdf/medicine update_2017/mu_095. [Last accessed on2020 Apr 02].

7. Fauci AS, Braunwald E, Isselbacher KJ, Wilson JD, Martin JB, Kasper DL, et al, editors. Harrison's Principles of Internal Medicine. 14th ed. New York: Mcgraw-Hill; 1997. p. 634-8, 672-8.

8. Diaz P, Vieira MA, Carneiro A, Fernandes N. A case of pancytopenia with many possible causes: How do you tell which is the right one? Eur J Case Rep Intern Med 2019;6:001012.

9. Pascutti MF, Erkelens MN, Nolte MA. Impact of viral infections on haematopoiesis: From beneficial to detrimental effects on bone marrow output. Front Immunol 2016;7:364.

10. Li MY, Li L, Zhang Y, Wang XS. Expression of the SARS-CoV-2 cell 
receptor gene ACE2 in a wide variety of human tissues. Infect Dis Poverty 2020;9:45

11. Clapes T, Lefkopoulos S, Trompouki E. Stress and non-stress roles of inflammatory signals during HSC emergence and maintenance. Front Immunol 2016;7:487.

12. Zhao Y, He J, Wang J, Li WM, Xu M, Yu X, et al. Development of pancytopenia in a patient with COVID-19. J Med Virol 2021;93:1219-20.

13. Francone M, Iafrate F, Masci GM, Coco S, Cilia F, Manganaro L, et al. Chest CT score in COVID-19 patients: Correlation with disease severity and short-term prognosis. Eur Radiol 2020;30:6808-17.

14. Hersby DS, Do TH, Gang AO, Nielsen TH. COVID-19-associated pancytopenia can be self-limiting and does not necessarily warrant bone marrow biopsy for the purposes of SARS-CoV-2 diagnostics. Ann Oncol 2021:32:121-3

Funding: None; Conflicts of Interest: None Stated.

How to cite this article: Shenoy V, Shekhar NC, Raj TS. A rare case report of transient pancytopenia in a patient with coronavirus disease 2019. Indian J Case Reports. 2021;7(7):274-277. 